\title{
The development of behavioral and endocrine abnormalities in rats after repeated exposure to direct and indirect stress
}

\author{
Willie Mark Uren Daniels' \\ Joachim de Klerk Uys' \\ Petra van Vuuren' \\ Daniel Joseph Stein ${ }^{2}$ \\ 'Division of Medical Physiology, \\ Faculty of Health Sciences, University \\ of Stellenbosch, South Africa; \\ ${ }^{2}$ Department of Psychiatry, Faculty of \\ Health Sciences, University of Cape \\ Town, South Africa
}

\begin{abstract}
The present study compared the effects of direct and indirect stress on the behavior and hypothalamic-pituitary-adrenal axis of rats. Animals were placed in a two compartment box. In one compartment the direct stressed rat was subjected to electric foot shocks randomly applied for 10 minutes $(0.5 \mathrm{~mA}$ of $1 \mathrm{~s}$ duration). In the adjacent compartment, the indirect stressed rats witnessed the application of these electric foot shocks. Our data showed substantial behavioral changes in the open field test, but limited effects in the elevated plus maze. The findings suggested that single and repeated stress exposure may have different consequences, that the effects of stress exposure may develop over time and persist for an extended period, and that both direct and indirect stressed rats displayed a hyposensitive HPA axis following acute restraint stress. Overall our observations moderately indicate direct exposure to elicit behavioral changes, and both direct and indirect exposure to stress to result in aberrations within the neuroendocrine system. With additional development our stress models may be considered for studying the complex interrelationship between an external stressor, and the experience of the organism.
\end{abstract}

Keywords: direct vs indirect stress, behavior, open field, elevated plus maze, HPA axis, stress response

\section{Introduction}

Stress is considered one of the major factors involved in the pathogenesis of affective disorders (McEwen 2000). Individuals are frequently subjected to stress and these stressors may be direct and/or indirect in nature. Examples of indirect stressors include indirect exposure to terrorist attacks (Schuster et al 2001; Stein et al 2004; Blanchard et al 2005; Bleich et al 2006), perceptions of lack of control of a stressful situation (Ozer et al 2003), or being subjected to subtle victimization (Estevez et al 2005). In addition the bouts of natural (Tsunami in Indonesia in 2004; Hurricanes Catherine and Rita in New Orleans in 2005), as well as man-made disasters (September 11 in New York, 2001; Bombing in London in 2005) have not only subjected a large number of persons directly to a traumatic event, but the widespread media coverage that followed, has also exposed masses of other individuals far away from the site, indirectly to the stressful situation. Clinical investigations studying the effects of scenarios such as these, reported a higher than normal prevalence of psychiatric symptoms within distant communities (Schuster et al 2001; Blanchard et al 2005). For instance partners of firefighters during the 1995 Oklahoma bombing, exhibited symptoms such as hyperarousal, intrusive re-experiencing, and heightened autonomic reactivity at 43-44 months after the event occurred (Pfefferbaum et al 2006). In a recent study Zimering and colleagues (2006) investigated the incidence of posttraumatic stress disorder (PTSD) in individuals exposed directly or indirectly to the collapsing of the 
World Trade Center. These authors found that both direct and indirect exposure to the disaster site led to PTSD. In spite of these interesting findings, it remains speculative as to whether the neurobiological mechanisms underpinning the eventual development of neuropathological disorders, are the same following direct or indirect exposure to severe stress.

The application of animal models has contributed substantially to our current understanding of the molecular mechanisms by which stressful events ultimately result in psychopathological states. For example, we have shown behavioral alterations following repeated stress exposure that are reminiscent of the abnormal behavioral patterns observed in patients afflicted with mood and anxiety disorders (Daniels et al 2000, 2004; Faure et al 2006; Uys et al 2006). However these studies, like others (Nico et al 2005; Joca et al 2006; Li et al 2006), often focus on the consequences of exposing test subjects directly to the stressor. The present study therefore employed methodologies to establish an animal model whereby the effects of indirect exposure to a stressor can be investigated. We subsequently compared the behavioral and endocrine effects in rats exposed to electrical foot shocks to that of rats that witnessed the application of these electrical foot shocks.

\section{Methods}

\section{Animals}

Male Sprague-Dawley rats from the Central Animal Facility of the University of Stellenbosch (AAALAC accredited) were used. The animals were reared under standard housing conditions at ambient temperatures of $22{ }^{\circ} \mathrm{C}$, humidity of $95 \%$ and a 12 hour day-night cycle (lights on at 0600 ; lights off at 1800). Food and water was freely available. The animals were transported to the experimental room 1 hour prior to the start of the experimental session. All experimental procedures were approved by the Ethics Committee for Experimental Animal Research of the University of Stellenbosch. The rats were exposed to one of three conditions: direct stress, indirect stress, or no-stress.

\section{Stress procedure}

The model used in this study is based on the methodology of Van den Berg and colleagues (1998). Briefly, two cage mate rats were placed in the two-compartment box for ten-minute sessions for one or five consecutive days. A perforated Perspex plate separated the two compartments to facilitate visual, auditory and olfactory communication. One session of direct stress consisted of 10 unpredicted electric foot shocks ( $0.5 \mathrm{~mA}$ for 1 second), delivered at random in one compartment (16 Channel Random Switch, Gentronics, South Africa). Random electrical currents were conducted through the metal grid floor of the compartment, generated by a shock generator and scrambler. To induce indirect stress, animals were placed in the adjacent compartment and witnessed the application of the electric foot shocks. These animals were therefore not given any electric foot shocks, but were exposed to visual, auditory and olfactory sensations from the direct stressed rats. Control animals were placed in pairs in the separate compartments, without receiving any shocks. The two-compartment box was cleaned with $70 \%$ ethanol after each pair of rats had been removed.

\section{Behavioral measures}

The Open field test was used to evaluate the behavior of the animals either 5 or 10 days after the last stress session. The animals were placed in the apparatus for five minutes and their activities recorded on video (Sony Digital Handy$\mathrm{cam}^{\circledR}$ ). Evaluators, blind to the status of the animals, rated the video recordings afterwards. Each animal was placed in the apparatus in the same position and the following behaviors were assessed in the open field: rearing, grooming, and time spent in the inner and outer zone of the open field. All four paws had to be over the demarcated lines to constitute a zone crossing.

A second test, the elevated plus maze, was used to validate the behavioral observations obtained from the open field test. Briefly the animals were placed in the neutral center square with its nose pointing towards an open arm. The behavior of the animals in the elevated plus maze were recorded for 5 minutes for scoring at a later stage. In addition to rearing and grooming, time spent in the open and closed arms, as well as the number of arm entries, were documented.

\section{Endocrine response}

In order to evaluate the integrity of the hypothalamicpituitary-adrenal axis following direct or indirect stress, rats were individually placed in a restrainer for 10 minutes and blood was collected before and 15 and 60 minutes post-stress for plasma corticosterone determinations using a Corticosterone Radio-immuno-assay kit (ImmuChem, Hamburg, Germany).

\section{Experimental protocols}

Experiment $A$ : rats were subjected to 1 -session of direct and indirect stress. 5 days after this stress session, open field and elevated plus maze activity was assessed for 5 minutes. 
Experiment B: rats were subjected to 5 sessions of direct and indirect stress, for 5 consecutive days. Again open field and elevated plus maze activity was assessed 5 days after the last stress session.

Experiment $C$ : rats were subjected to 5 sessions of direct and indirect stress for 5 consecutive days, and also subjected to an additional 10 minutes of restraint stress 5 days after the last stress session. In order to evaluate HPA axis activity trunk blood was collected at 0,15 and 60 minutes post-restraint stress.

Experiment D: rats were subjected to 5 sessions of direct and indirect stress for 5 consecutive days. However, in this experiment the open field and elevated plus maze activity was assessed 10 days after the last stress session.

\section{Statistical analysis}

All data are presented as mean \pm SEM. The Kruskal Wallis test was used to identify significant differences within experiments and Dunn's multiple Comparison post-test was used to assess the significance of differences between experimental groups with $\mathrm{p} \leq 0.05$ considered as significant. The computer package GraphPad Prism version 4.0 (San Diego, California) was used for this purpose.

\section{Results}

In experiment $\mathrm{A}$, rats were exposed to 1 stress session and their behavior evaluated 5 days later using the open field test and the elevated plus maze. A significant increase in grooming by rats exposed to direct stress was observed in the open field in comparison to the indirect stressed animals and control group (Figure 1B: Kruskal Wallis test $\mathrm{p}=0.012$; Dunn's Multiple Comparison post-test $\mathrm{p}<0.01$ ). There was no difference between the frequencies of grooming behaviors in the indirect group compared with the controls. All other parameters investigated in the open field test were found to be nonsignificant between the 3 groups of animals. Subsequent assessment of the behavior of the various groups on the elevated plus maze also yielded no significant differences in any of the parameters measured (Figure 2).

In experiment $\mathrm{B}$, increasing the number of stress sessions from 1 to 5 resulted in a significant reduction in rearing behavior in direct stressed rats compared with both indirect stressed and control animals (Figure 3: Kruskal Wallis test $\mathrm{p}=0.0035$, Dunn's Multiple Comparison post-test $\mathrm{p}<0.01$ for Direct vs Indirect and $\mathrm{p}<0.05$ for Direct vs Control). However, no significant differences were observed between the 3 groups of animals in terms of grooming behavior, or any of the other parameters in the open field. When the behavior of these animals was evaluated on the elevated plus maze, the differences between the various parameters were found to be nonsignificant (Figure 4).

In experiment $\mathrm{C}$, the HPA axis was evaluated in rats exposed to 5 sessions of stress, by subjecting the animals to an additional bout of restraint stress. There was no significant difference between the baseline plasma corticosterone levels of any of the groups tested. Whilst the concentration in corticosterone increased significantly in all 3 groups 15 minutes after the restraint stress, this increase was significantly diminished in both the direct and the indirect stressed groups when compared to controls (Figure 5: Kruskal Wallis Test $\mathrm{p}=0.0142$, Dunn's Multiple Comparison test $\mathrm{p}<0.05$ for both Direct vs Control as well as Indirect vs Control ). At 60 minutes, the plasma corticosterone concentrations of both stressed groups were significantly higher than controls (Figure 5: Kruskal Wallis Test $\mathrm{p}=0.00$ 178, Dunn's Multiple Comparison test $\mathrm{p}<0.05$ for both Direct vs Control as well as Indirect vs Control).

In experiment $\mathrm{D}$, when the behavior of another group of animals that were stressed for 5 days, was evaluated with the open field test 10 days after the exposure to the last stress, a number of remarkable findings were observed in the open field test (Figure 6). Direct stressed animals had significantly lower scores than control rats in rearing (Figure 6A: Kruskal Wallis test $\mathrm{p}=0.0015$, Dunn's Multiple Comparison Test $\mathrm{p}<0.001$ ), percentage time in the inner and outer zone (Figures 6C and 6D: Kruskal Wallis test $\mathrm{p}=0.0295$, Dunn's Multiple Comparison Test $\mathrm{p}<0.05$ ), and the number of blocks crossed (Figure 6E: Kruskal Wallis test $\mathrm{p}=0.0004$, Dunn's Multiple Comparison Test $\mathrm{p}<0.01$ for both Direct vs Indirect as well as Direct vs Control). Animals subjected to indirect stress also displayed reduced scores in these parameters when compared to the control group, but this reduction did not reach statistical significance. The only parameter that was found to be significantly different on the elevated plus maze, was the number of entries into the open arms. Animals exposed to both direct and indirect stress displayed significantly less entries when compared to controls (Figure 7E: Kruskal Wallis test $\mathrm{p}=0.0155$, Dunn's Multiple Comparison Test $\mathrm{p}<0.01$ ).

\section{Discussion The effects of direct and indirect stress on behavior}

One of the most widely used tests in behavioral research to evaluate environmental manipulations on the emotionality of rodents, is the open field test (Crawley 1985; Prut and Belzung 2003), although its traditional use has been questioned recently (Ennaceur et al 2006). Open field methods 
A: Open Field 1d stress; behavior at 5d Rearing

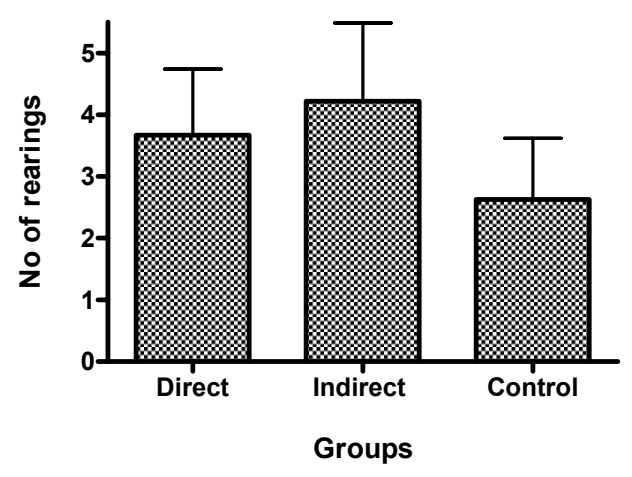

C: Open Field 1d stress; behavior at $5 d$ Time in inner zone

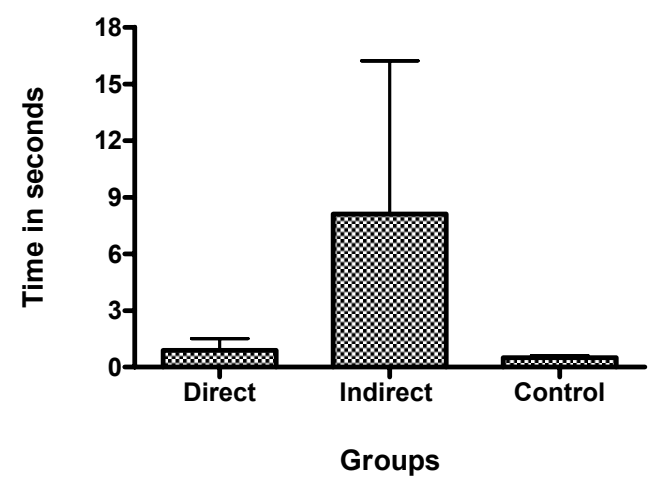

B: Open Field 1d stress; behavior at $5 d$ Grooming

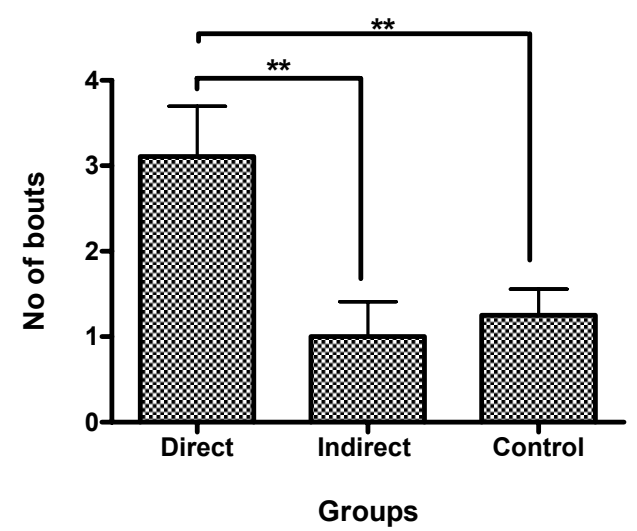

D: Open Field 1d stress; behavior at 5d Time in outer zone

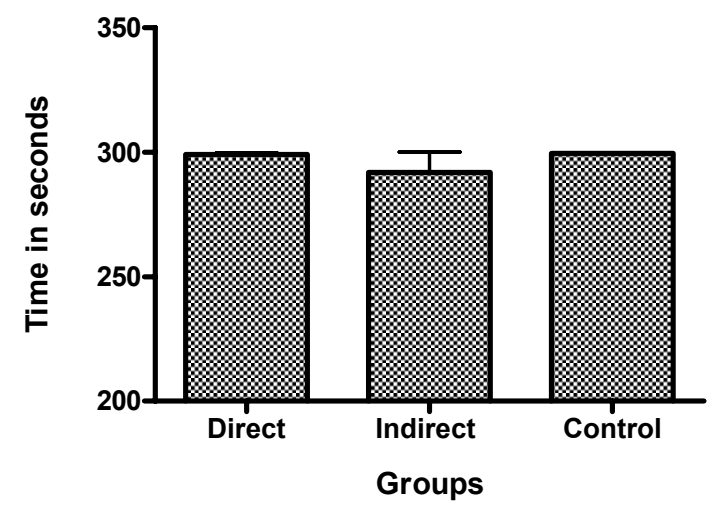

\section{E: Open Field 1d stress; behavior at 5d} Number of blocks crossed

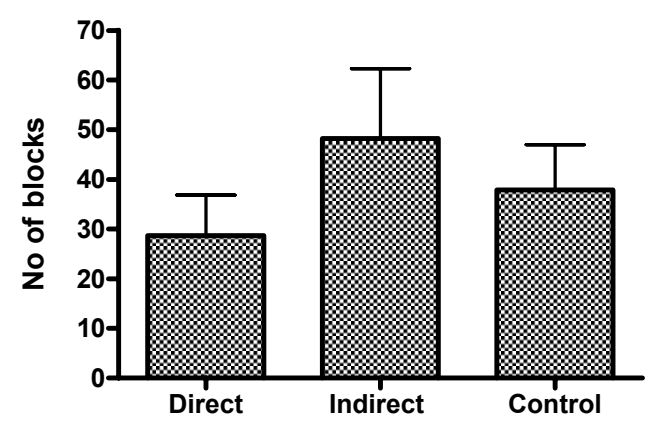

Figure I Animals were subjected to a single session of direct $(n=9)$, indirect $(n=9)$ stress, or no stress $($ control, $n=8)$ and behavior was assessed 5 days later during the Open Field test (Experiment $A$ in text). Values are shown as means \pm SEM.

Notes: ${ }^{* *} \mathrm{p} \leq 0.0$ I; Significantly different from direct stressed group (Dunn's Multiple Comparison test). 
A: EPM 1d stress; behavior at 5d Rearing

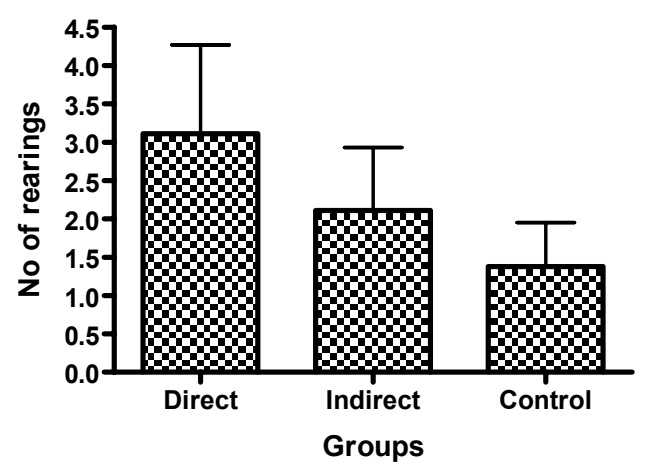

C: EPM 1d stress; behavior at 5d Time in open arms

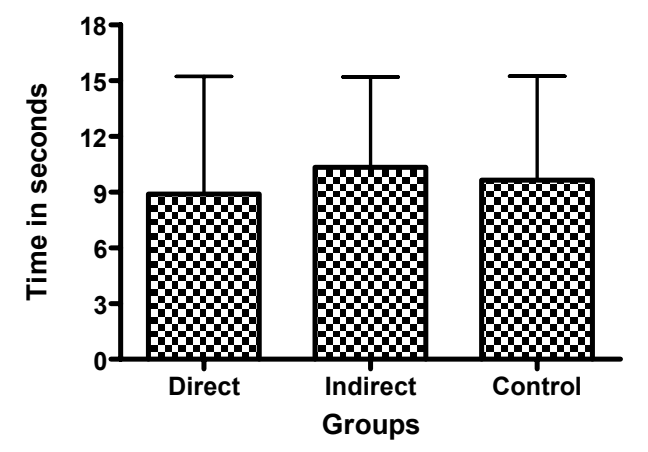

E: EPM 1d stress; behavior at 5d Entries in open arms

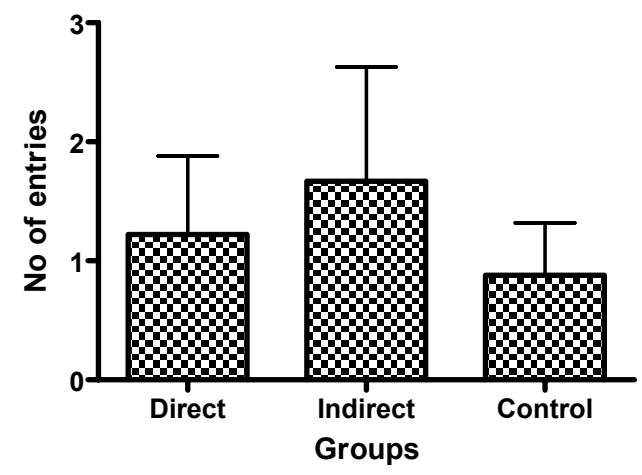

B: EPM 1d stress; behavior at 5d Grooming

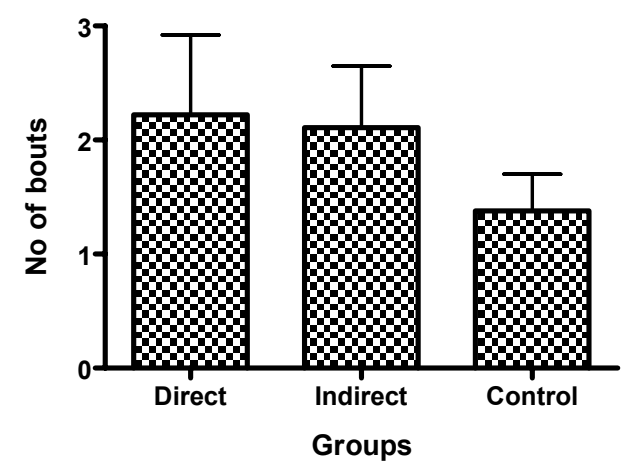

D: EPM 1d stress; behavior at 5d Time in closed arms

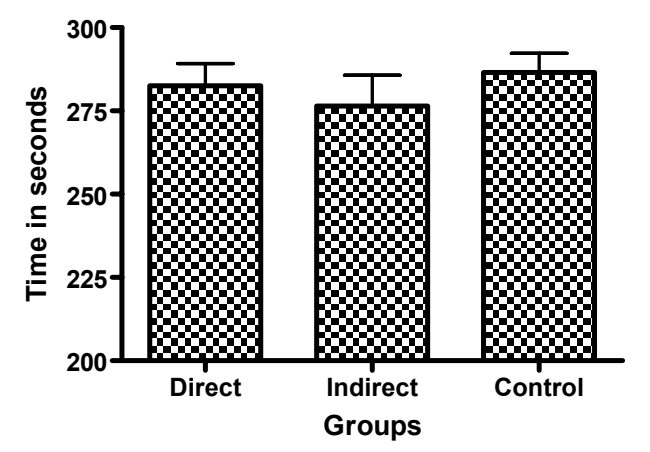

\section{F: EPM 1d stress; behavior at 5d Entries in closed arms}

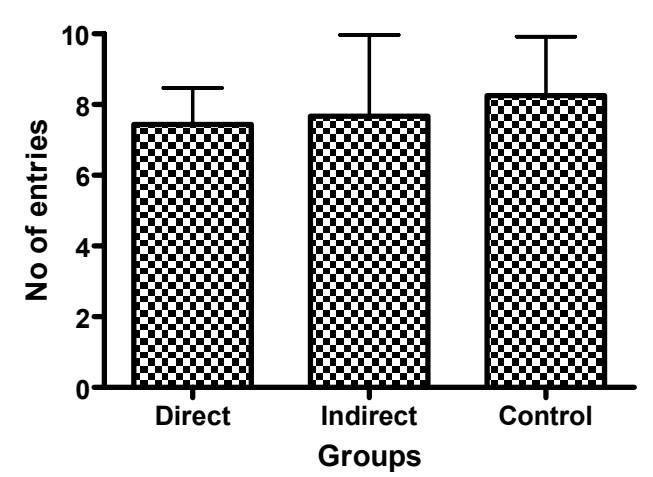

Figure 2 Animals were subjected to a single session of direct $(n=9)$, indirect $(n=9)$ stress, or no stress (control, $n=8)$ and behavior was assessed 5 days later on the elevated plus maze (Experiment $A$ in text). Values are shown as means \pm SEM. 
A: Open Field $5 d$ stress; behavior at $5 d$ Rearing

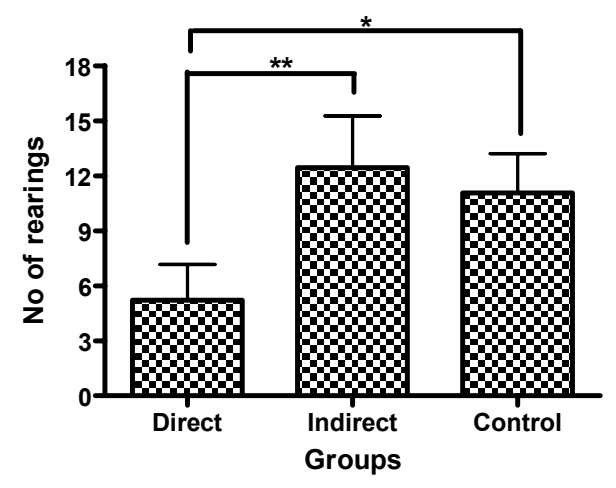

C: Open Field 5d stress; behavior at 5d Time in inner zone

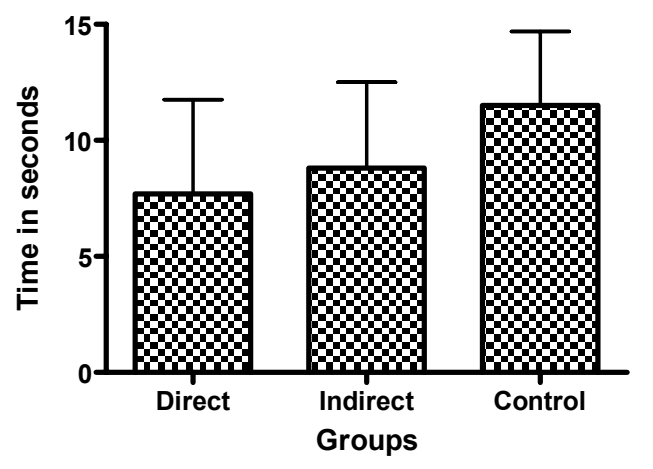

B: Open Field 5d stress; behavior at $5 d$ Grooming

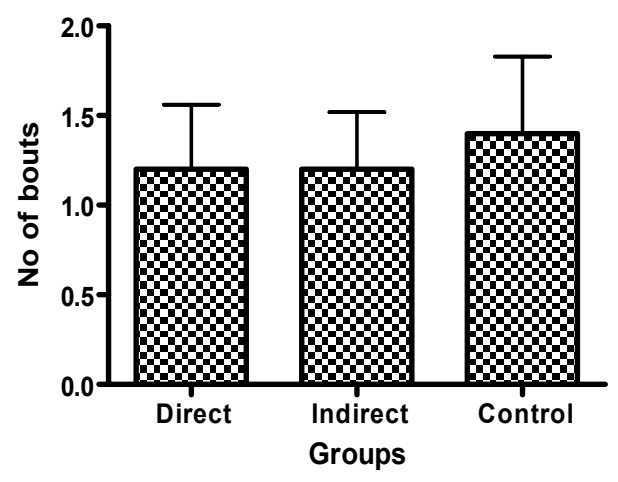

D: Open Field $5 d$ stress; behavior at $5 d$ Time in outer zone

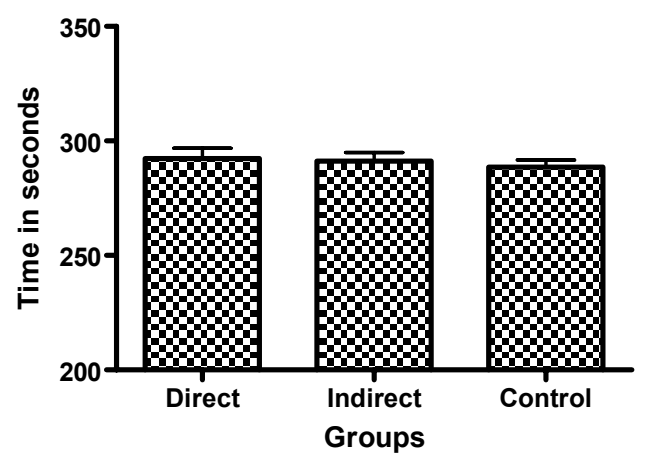

E: Open Field 5d stress; behavior at 5d Number of blocks crossed

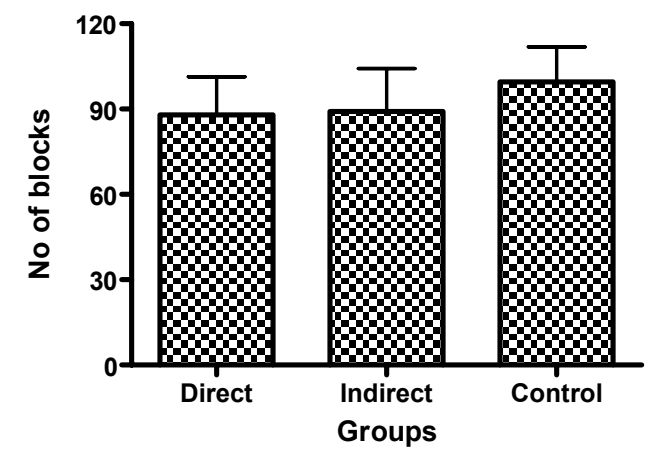

Figure 3 Animals were subjected to 5 days of direct $(n=10)$, or indirect $(n=10)$ stress, or no stress $(n=10)$ and their behavior evaluated 5 days later during the open field test (Experiment $B$ in text). Values as shown as means \pm SEM.

Notes: ${ }^{*}<<0.05 ;{ }^{* *} p<0.01$; Both significantly different direct group respectively (Dunn's Multiple Comparison test). 


\section{A: EPM 5d stress; behavior at 5d Rearing}

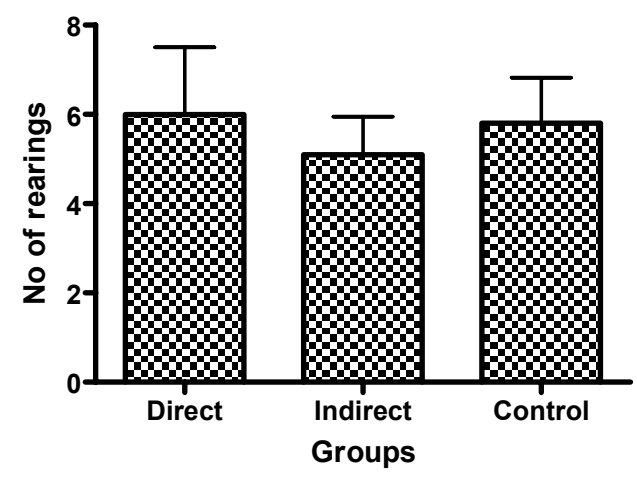

\section{C: EPM 5d stress; behavior at 5d} Time in open arms

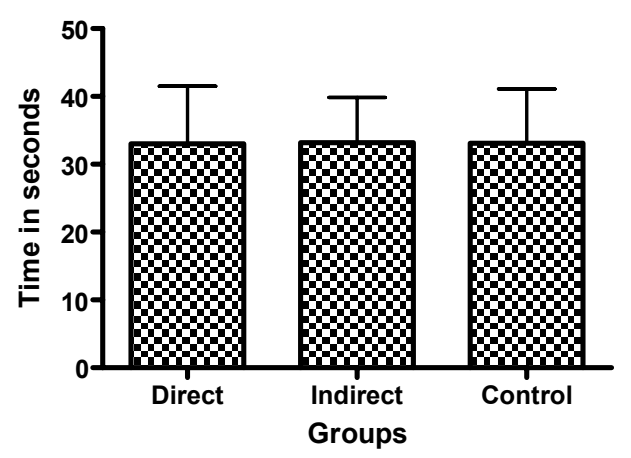

E: EPM 5d stress; behavior at 5d Entries in open arms

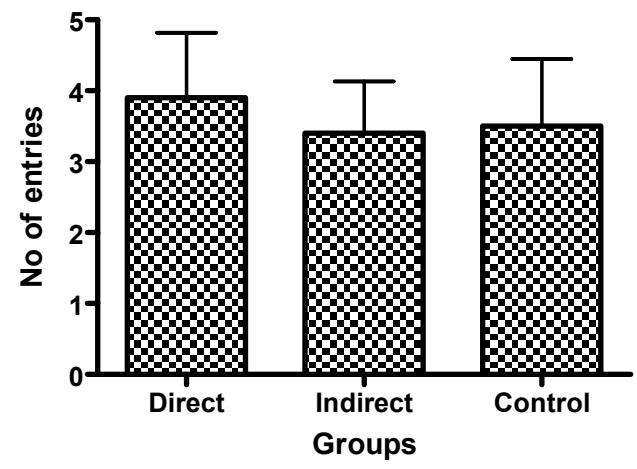

\section{B: EPM 5d stress; behavior at 5d Grooming}

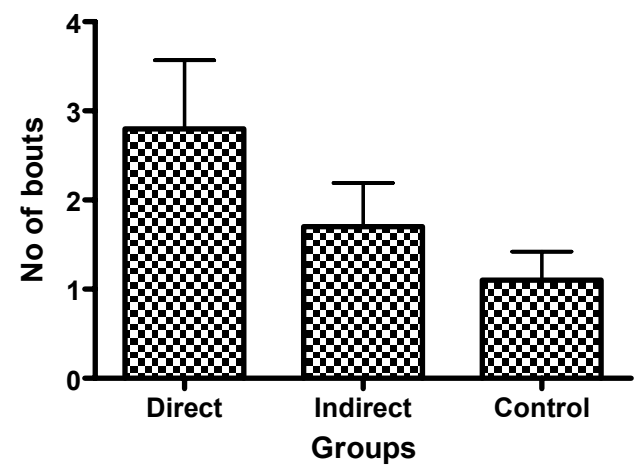

D: EPM 5d stress; behavior at 5d Time in closed arms

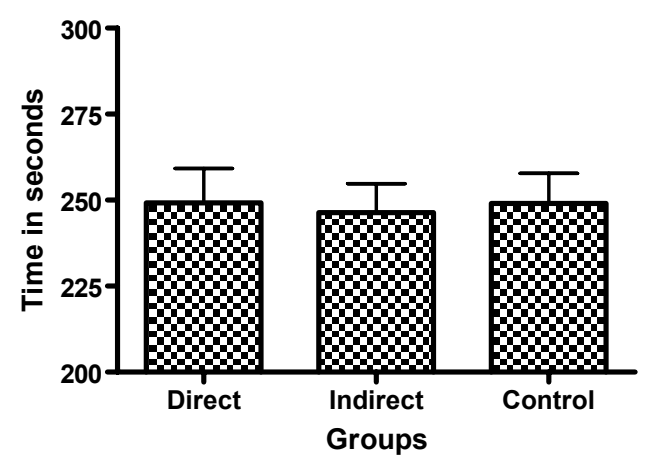

\section{F: EPM 5d stress; behavior at 5d} Entries in closed arms

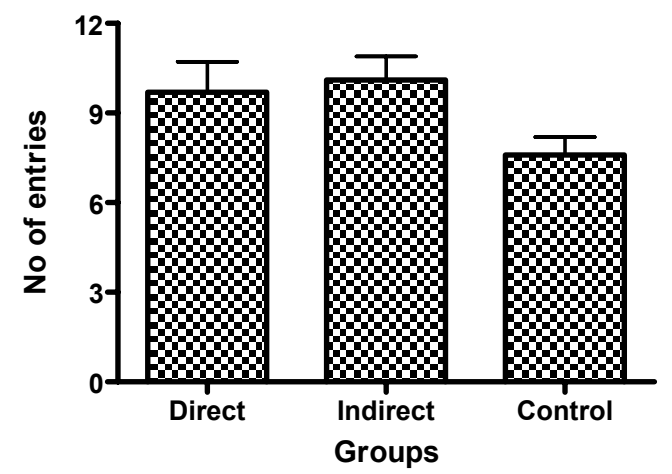

Figure 4 Animals were subjected to 5 days of direct $(n=10)$, or indirect $(n=10)$ stress, or no stress $(n=10)$ and their behavior evaluated 5 days later on the elevated plus maze (Experiment $B$ in text). Values as shown as means \pm SEM. 


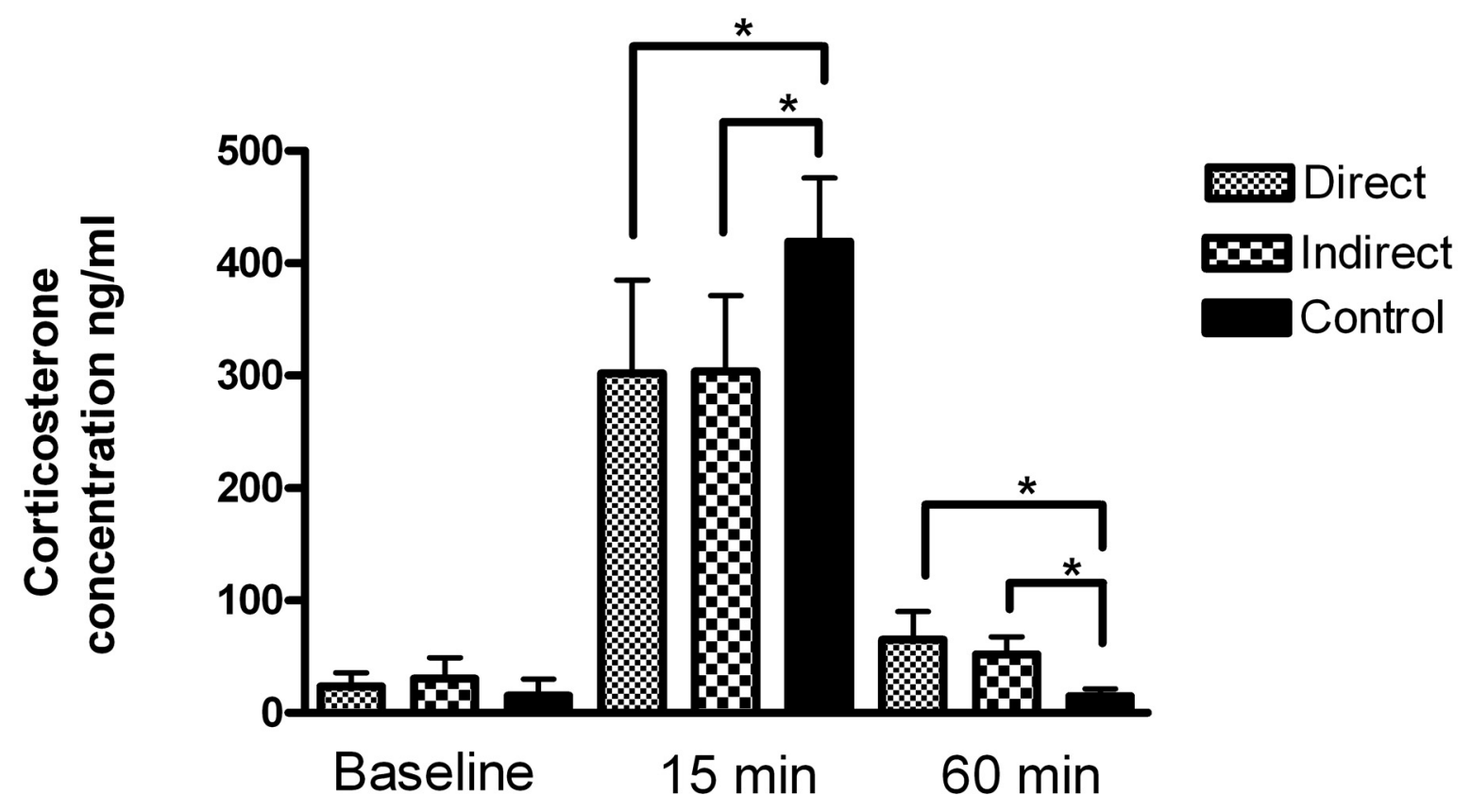

Figure 5 Rats were exposed to the 5 -day stress protocol (Experiment $\mathrm{C}$ in text). After a further 5 days the animals were subjected to acute restraint stress. Trunk blood was collected and corticosterone concentrations were measured at baseline $(n=10$ per group), 15 minutes $(n=8$ per group), and 60 minutes ( $n=7$ per group) after the restraint stress. Values are shown as means \pm SEM.

Notes: ${ }^{*} \mathrm{p}<0.01$; Significantly different from the 15 and 60 minutes corticosterone concentration of the respective control group (Dunn's Multiple Comparison test).

not only allow for easy quantification of aspects of locomotor behavior but also exploratory behavior, curiosity, and coping. Therefore behavioral parameters such as grooming, rearing, and time spent in the inner and outer zone were noted. In addition we evaluated the animals' behavior using the elevated plus maze. This instrument has also been thoroughly validated as an extremely useful tool to assess the emotional status of rodents (Pellow et al 1985; Rodgers and Dalvi 1997).

Overall the various observations of the open field test and the elevated plus maze reflected only moderate changes in behavior. Nevertheless there were some interesting findings suggesting that duration of exposure, as well as type of stress (direct or indirect) may lead to different phenomenological and biological changes. The data from the open field showed that exposure to a single session of stress can lead to behavioral abnormalities depending on the type of stress. Grooming behavior occurs naturally and has a diverse, patterned structure, particularly when evoked under predictable conditions. Increased grooming is known to be triggered by environmental disturbances, such as conditions of stress that include handling, restraint, and novelty (Komorowska and Pellis 2004; Kalueff and Tuohimaa 2005). In our investigation direct stressed rats, in the single stress experiment, groomed excessively in comparison with the indirect stressed rat as well as the control rats. This result suggested that grooming may be directly related to the strength of the stressor. However we are aware that this may not always be the case. According to a study done by Van Erp and colleagues (1994), rats used as intruders in the territory of another rat, maintained a constant low level of grooming. In contrast, rats placed in a novel cage steadily increased grooming during the observation period. These observations therefore indicate that grooming cannot simply be understood as an immediate response required to reduce the level of arousal following stressors. Instead following exposure to a stressor, grooming may be seen as a self-soothing behavior that assists in coping with the stressful situation.

It has long been recognized that the interaction of grooming and anxiety is rather complex and that rat grooming can be increased in both high and low stress situations. For example, after anxiolytic and anxiogenic drugs, both activation and inhibition of grooming was observed (File and Baldwin 1987). Grooming activity is therefore also considered a mechanism serving to alleviate anxiety, ie, as a displacement activity in rats. It is well-known that animals 
A: Open Field 5d stress; behavior at $10 \mathrm{~d}$
Rearing

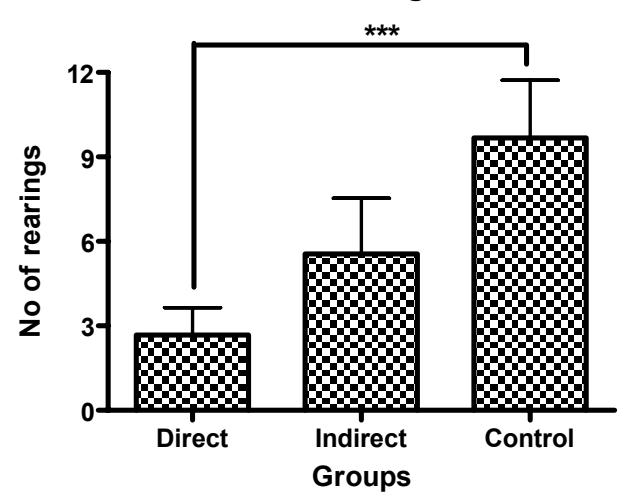

C: Open Field $5 d$ stress; behavior at $10 d$ Time in inner zone

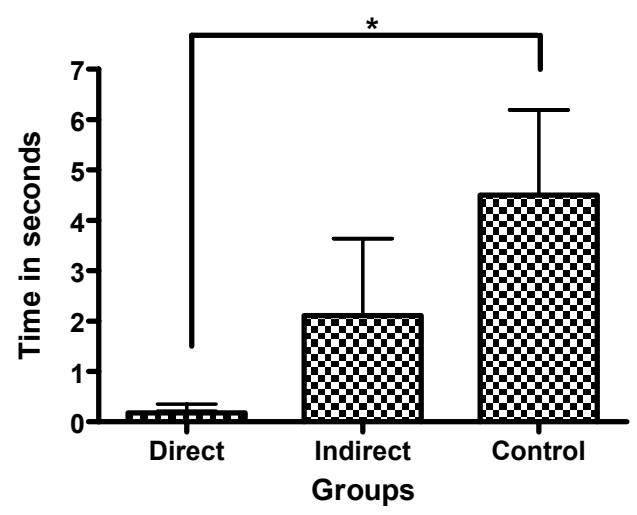

B: Open Field 5d stress; behavior at 10d Grooming

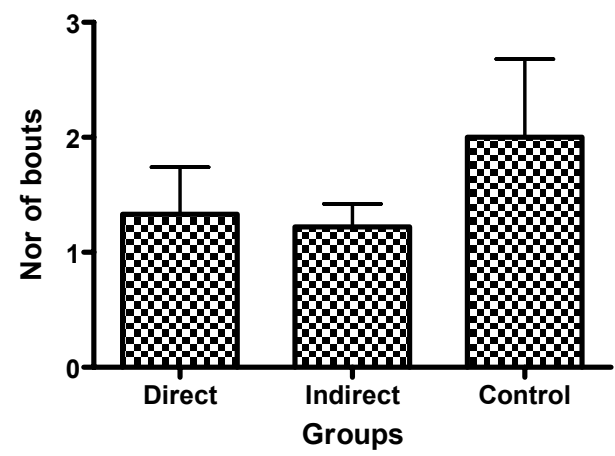

D: Open Field $5 \mathrm{~d}$ stress; behavior at $10 \mathrm{~d}$ Time in outer zone

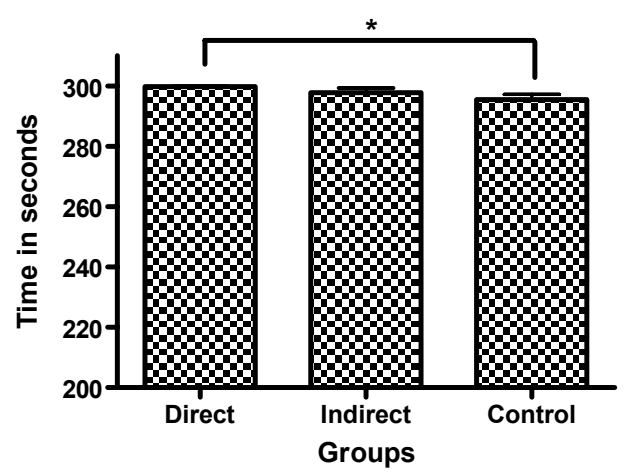

E: Open Field $5 \mathrm{~d}$ stress; behavior at $10 \mathrm{~d}$ Number of blocks crossed

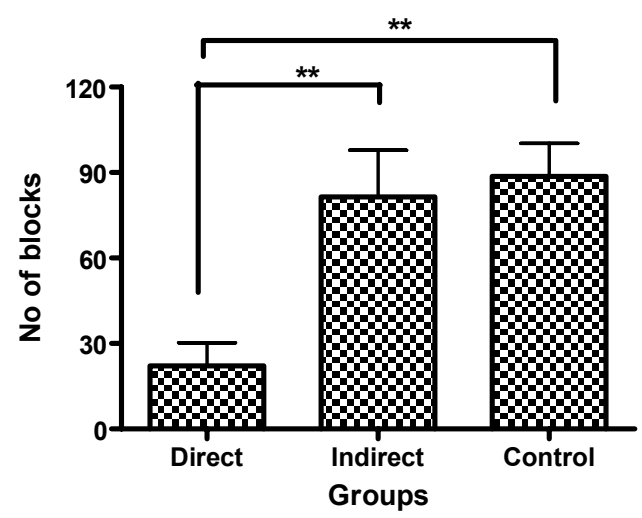

Figure 6 Animals were subjected to 5 days of direct $(n=9)$, indirect $(n=9)$ stress, or no stress $(n=6)$ and their behavior evaluated 10 days later during the open field test (Experiment $D$ in text). Values as shown as means \pm SEM.

Notes: ${ }^{*} p<0.05$; Significantly different from direct stressed group (Dunn's Multiple Comparison test); **p $<0.01$; Significantly different from direct stressed group (Dunn's Multiple Comparison test); ${ }^{* * *} \mathrm{p}<0.00$ I; Significantly different from direct stressed group (Dunn's Multiple Comparison test). 
A: EPM 5d stress; behavior at 10d

Rearing

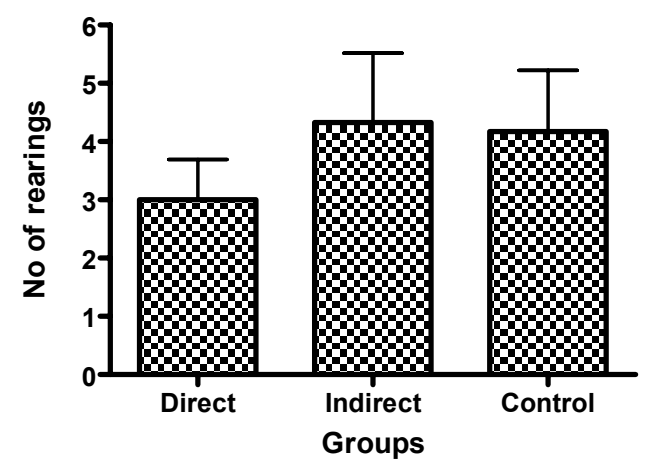

C: EPM 5d stress; behavior at $10 d$ Time in open arms

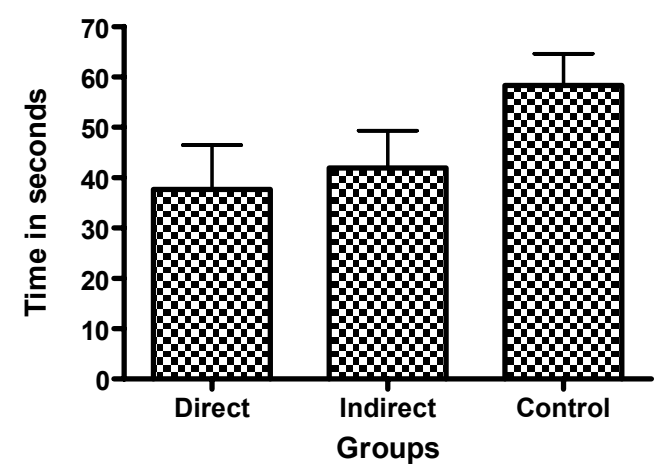

E: EPM 5d stress; behavior at 10d Entries in open arms

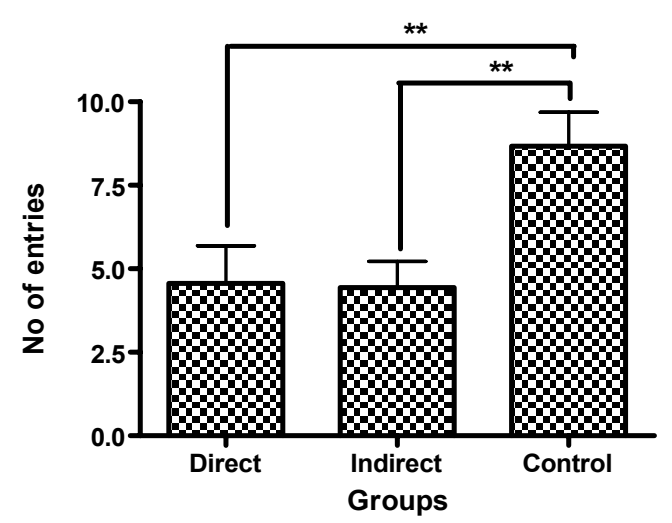

B: EPM 5d stress; behavior at 10d Grooming

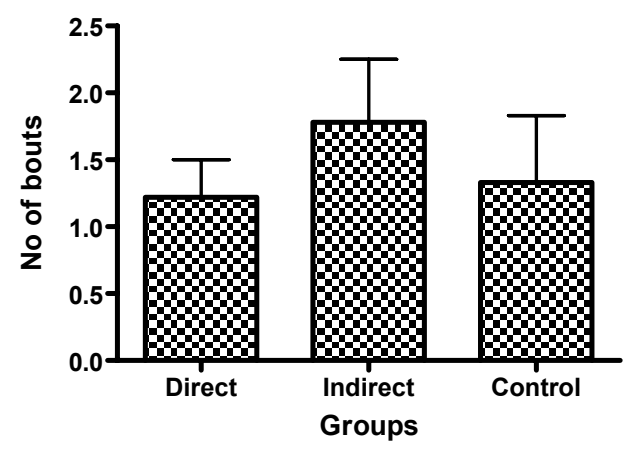

D: EPM 5d stress; behavior at 10d Time in closed arm

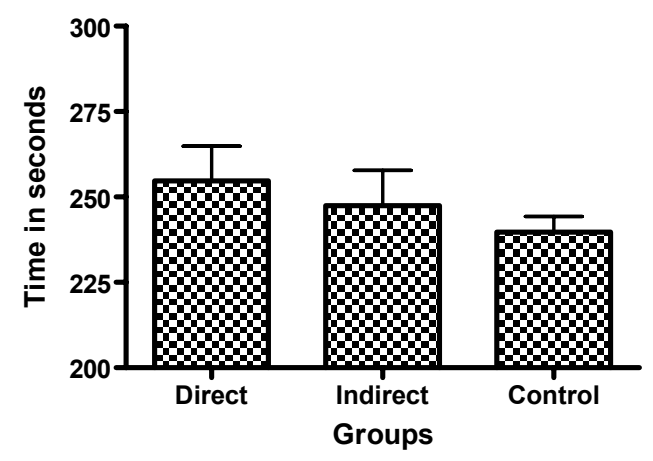

\section{F: EPM 5d stress; behavior at 10d Entries in closed arms}

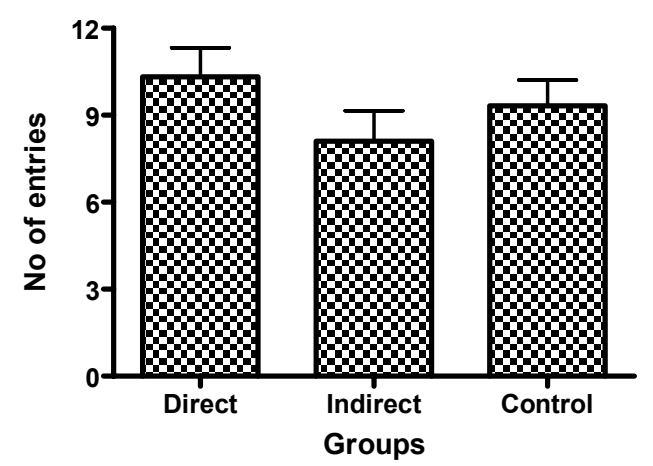

Figure 7 Animals were subjected to 5 days of direct $(n=9)$, indirect $(n=9)$ stress, or no stress $(n=6)$ and their behavior evaluated 10 days later on the elevated plus maze (Experiment $D$ in text). Values as shown as means \pm SEM.

Notes: **p $<0.01$; Significantly different from control (Dunn's Multiple Comparison test). 
often make extensive use of displacement activity, such as freezing behavior and/or jumping, as a strategy to cope with stress (Kualeff and Tuohimaa 2005).

A second interesting finding on the open field was the substantial difference in behavior obtained when the rats were exposed to repeated stress as opposed to a single bout of stress assessed 5 days after the last stress session. Comparing single to repetitive stress, directly stressed rats showed reduced rates in rearing, while the indirect stressed rats showed similar levels as control animals. Low levels of rearing in the open field test may reflect high emotionality and fearfulness (Van den Berg et al 1998), and anxious behavior (Carneiro et al 2005). Rearing is a component of exploratory behavior and when displayed in an open field, the behavior may be multi-factorial. With exploration and stress being the main motivational systems underlying rearing behavior (Crusio 2005), variations observed in its expression may therefore be due to differences in either of these two factors. Increases in rearing behavior may also reflect other advantages. When animals are under threat or are attracted by novel stimuli, increased exploration may in fact improve the chances of finding life necessities (food) or promote survival (finding shelter or escape routes).

Similar to grooming, interpretation of rearing data alone may be problematic. In our search to obtain a better understanding of the effects of direct stress versus indirect stress, rats were subjected to stress repeatedly for 5 days and assessed 10 days later. At this time point animals subjected to direct stress showed significant behavioral abnormalities in the open field test with respect to their emotional status. These animals had reduced rates in rearing and spent significantly less time in the inner zone when compared to both the indirect stressed and control groups. This observation was comparable to experiments where uncontrollable electric foot shocks caused decreased swimming activity in mice (Prince and Anisman 1984). Animals that received direct stress also displayed decreased locomotor activity as indicated by diminished block crossings in the open field, suggesting the development of hypoactivity. Interestingly both the direct and indirect stress groups of animals showed reduced entries into the open arms of the elevated plus maze. While this single observation may be inadequate to define the mood status of the animals, it does together with the open field result, suggest a change in activity level of the animals. Some support for this finding comes from studies by Estanislau and Morato (2005), where rats that were in-utero exposed to indirect stress (their dams were subjected to electric foot shock) displayed a reduction in exploration of the open arms of an elevated plus maze. In another experiment Van der Hart et al (2005) demonstrated how tree shrews, subjected to a 7 day period of psychosocial stress, also displayed decreased locomotor activity. These studies show an association between prolonged stress, decreased locomotor activity and an increased state of anxiety, and our results provide some evidence (although limited) that may support this concept in both the direct and indirect stressed groups.

To some degree the present data reflect a development of behavioral deficits over time. There was a distinction between the observed behavioral abnormalities in animals when assessed 10 days rather than 1 or 5 days after stress exposure, particularly in the open field test. The notion of the development and then persistence of abnormal behavior following an adverse event is supported by clinical studies. For instance some studies have provided evidence showing adverse experiences during childhood to be a major contributory factor to psychopathology later in life. For example, sexually abused girls developed depression and/or anxiety-related disorders as adults (De Bellis et al 1994; Heim et al 2000; Heim and Nemeroff 2001), while post-traumatic stress disorder in war veterans has been diagnosed long after the termination of the battle (Hilsenroth et al 2005).

\section{The effects of direct and indirect stress on the endocrine response}

One of the dominant parts of the stress response is neuroendocrine activation. Therefore studying the functioning of the HPA axis is of importance in our quest to characterize the consequences of direct and indirect stress. Two approaches were adopted in the present experiments to provide us with information about the effects of direct and indirect stress on the HPA axis: 1) baseline corticosterone concentrations were determined to indicate basal HPA axis activity, and 2) rats were subjected to acute restraint stress and the corticosterone concentrations were measured at time points 15 and 60 minutes post stress, to reflect HPA axis responsivity. Basically our data showed no significant difference in any of the groups studied with respect to their basal plasma corticosterone levels. However both direct and indirect stressed rats displayed a blunting of the stress response following acute restraint stress, as well as a delay in the return to pre-stress levels.

The fact that there were no significant differences in baseline corticosterone concentrations between any of the groups of animals, suggested that the normal circadian activity of the HPA axis remained intact despite being subjected to the various stress protocols. This is not surprising as a number of 
basic studies have reported normal basal corticosterone levels in rats exposed to electric foot shock stress. For example in two recent studies rats were subjected repeatedly to inescapable foot shock and these authors also observed no changes in baseline corticosterone levels (Helmreich et al 2005; Louvart et al 2005). Even in clinical studies, while many psychiatric disorders are to be associated with abnormalities in HPA axis regulation (Nemeroff 2004), others show no difference in basal cortisol concentrations between patients and controls (Bremner et al 2003; Burke et al 2005). It is obvious that basal plasma glucocorticoid levels as a parameter of HPA axis activity remain controversial.

In contrast to the basal concentrations, both direct stressed and indirect stressed animals showed diminished levels of corticosterone 15 minutes after acute restraint stress. This reduced corticosterone secretion indicated a blunting of HPA axis responsivity to mediators of stress. This result is in accordance with earlier studies from our laboratory where postnatal maternal separation also led to a hypo-responsive HPA axis (Daniels et al 2004). Interestingly, Pijlman (2001) showed direct and indirect stressed rats to have a differential endocrine response. Direct stressed animals showed increased corticosterone levels compared to controls, while indirect stressed animals had the same stress response as the control animals. Discrepancies in this data and ours may derive from differences in the respective experimental protocols. These include the use of different rat strains, variations in current strength and timing of application during the foot shocks, as well as dissimilarities in time after stress exposure when blood was collected for the determination of hormone concentrations.

According to McEwen (2000), acute stress is frequently followed by an activation of a delayed hypersensitivity response and this is related to the magnitude of glucocorticoid secretion. Chronic stress, on the other hand, produces a suppression of the delayed hypersensitivity and also suppresses the initial sensitization of the response. Such a suppressive effect may cause the blunting HPA axis response observed in our experiments. In support for this reasoning is Antelman's hypothesis (1994) that the stress-restress paradigm may produce sensitization in some biological systems, in this case, inhibitory elements of the HPA axis, to yield abnormal corticosterone secretion. Upregulation of hippocampal glucocorticoid receptors that function to inhibit HPA axis activity may therefore be involved in this process (Liberzon et al 1997).

Experiments by Johnson and colleagues (1992) showed that during constant stress, the effectors of the generalized stress response (including the HPA axis) interfered with, rather than promote the animal's ability to adapt to the stressful situation. However unpredictability of the stressor seemed to be a critical factor in this inability to habituate. In addition, in animal models of chronic stress, the acute stress response often displays signs of habituation when the stressor is presented repeatedly (Jodar et al 1996). It may therefore be possible that the random delivery of foot shocks used in our experimental repeated stress paradigm could have partially prevented similar adaptive responses, and hence no changes in baseline corticosterone levels but significant differences in HPA axis response to restraint stress.

Plasma corticosterone inhibits corticosterone-releasingfactor and adreno-corticotropic-hormone secretion under normal conditions of acute stress. Under chronic stress this negative feedback inhibitory effect may have been amplified resulting in a down-regulation of corticosterone-releasing-factor and adreno-corticotropic-hormone release upon acute stress. This explanation is plausible as patients suffering from PTSD exhibit a hypersensitivity of the HPA axis to dexamethasone inhibition (Yehuda et al 1993). According to van Dijken and colleagues (1993) increased release of vasopressin may underlie the sensitization of the hypothalamic-pituitary-adrenal axis, since vasopressin stores were found to be increased in the external stores of the median eminence where fibers of the corticotropin-releasing-hormone neurons are located. Stressful stimuli are known to specifically upregulate vasopressin RNA in these corticotropin-releasing-hormones and vasopressin can potentiate the corticotropin-releasing-hormone induced release of adrenocorticotropic-hormone in the pituitary (Van Dijken et al 1992, 1993).

\section{Summary}

The focus of the present study was to investigate behavioral and endocrine responses in rats subjected to direct and indirect stress. Whilst the open field test indicated substantial behavioral abnormalities in stressed animals, these findings were not supported by our results from the elevated plus maze. Caution is therefore required when conclusions are to drawn. Nevertheless our data do seem to suggest a difference in behavior between the group of animals that received direct stress and controls. In contrast the difference in behavior between the indirect and control groups was negligible. The neuroendocrine data does however points to some neurobiological disturbance in the indirect stressed group, suggesting that it may be worthwhile to explore the models used in the present study further to elucidate the complexities associated with direct and/or indirect exposure to stress. 


\section{Acknowledgments}

This research was supported by the National Research Foundation and the Medical Research Council of South Africa, and formed part of the M.Sc thesis of Ms P van Vuuren.

\section{References}

Blanchard EB, Rowell D, Kuhn E, et al. 2005. Posttraumatic stress and depressive symptoms in a college population one year after the September 11 attacks: the effect of proximity. Behav Res Ther, 43:143-50.

Bleich A, Gelkopf M, Melamed Y, et al. 2006. Mental health and resiliency following 44 months of terrorism: a survey of an Israeli national representative sample. $B M C M e d, 4: 21-32$.

Bremner JD, Vythilingam M, Anderson G, et al. 2003. Assessment of the hypothalamic-pituitary-adrenal axis over a 24-hour diurnal period and in repsonse to neuroendocrine challenges in women with and without childhood sexual abuse and posttraumatic stress disorder. Biol Psychiatry, 54:710-18.

Burke HM, Davis MC, Otte C, et al. 2005. Depression and cortisol responses to indirect stress: a meta-analysis. Psychoneuroendocrin, 30:846-56.

Carneiro LM, Diogenes JP, Vasconcelos SM, et al. 2005. Behavioral and neurochemical effects on rat offspring after prenatal exposure to ethanol. Neurotoxicol, 27:585-92.

Crawley JN. 1985. Exploratory behavior models of anxiety in mice. Neurosci Biobehav Rev, 9:37-44.

Crusio WE, Schwegler H. 2005. Learning spatial orientation tasks in the radial-maze and structural variation in the hippocampus in inbred mice. Behav Brain Functions, 1:3-14.

Daniels WM, Pietersen CY, Carstens ME, et al. 2000. Overcrowding induces anxiety and causes loss of serotonin 1A receptors in rats. Metab Brain Dis, 15:287-95.

Daniels WM, Pietersen CY, Carstens ME, et al. 2004. Maternal separation in rats leads to anxiety-like behavior and a blunted ACTH response and altered neurotransmitter levels in response to a subsequent stressor. Metab Brain Dis, 19(1-2):3-14.

De Bellis MD, Lefter L, Trickett PK, et al. 1994. Urinary catecholamine excretion in sexually abused girls. J Am Acad Child Adolescence Psychiatry, 33:320-7.

Ennaceur A, Michalikova S, Chazot PL. 2006. Models of anxiety: responses of rats to novelty in an open space and an enclosed space. Behav Brain Res, 171:26-49.

Estanislau C, Morato S. 2005. Prenatal stress produces more behavioral alterations than maternal separation in the elevated plus-maze and in the elevated T-maze. Behav Brain Res, 163:70-7.

Estevez E, Musitu G, Herrero J. 2005. The influence of violent behavior and victimization at school on indirect distress: the role of parents and teachers. Adolescence, 40:183-96.

Faure J, Uys JD, Marais L, et al. 2006. Early maternal separation followed by later stressors leads to dysregulation of the HPA axis and increases in hippocampal NGF and NT-3 levels in a rat model. Metab Brain Dis, 21(2-3):181-8.

File SE, Baldwin HA. 1987. Effects of beta-carbolines in animal models of anxiety. Brain Res Bull, 19:293-9.

Heim C, Nemeroff CB. 2001. The role of childhood trauma in the neurobiology of mood and anxiety disorders: preclinical and clinical studies. Biol Psychiatry, 49:1023-39.

Heim C, Newport DJ, Heit S, et al. 2000. Pituitary-adrenal and autonomic responses to stress in women after sexual an direct abuse in childhood. JAMA. 284:592-7.

Helmreich DL, Parfitt DB, Lu XY, et al. 2005. Relation between the hypothalamic-pituitary-thyroid (HPT) axis and the hypothalamic-pituitaryadrenal (HPA) axis during repeated stress. Neuroendocrin, 81:183-92.

Hilsenroth M, Arsenault L, Sloan P. 2005. Assessment of combat-related stress and direct symptoms of Gulf War veterans: criterion validity of selected hand test variables. J Personality Assessment, 84:155-2.
Joca SR, Zanelati T, Guimaraes FS. 2006. Post-stress facilitation of serotonergic, but not noradrenergic, neurotransmission in the dorsal hippocampus prevents learned helplessness development in rats. Brain Res, 1087:67-74.

Jodar L, Takahasi M, Kaneto H. 1996. FS stress induces long-lasting memory facilitation: involvement of cholinergic pathways. Pharmacol Biochem Behav, 53:735-40.

Johnson EO, Kamilaris TC, Chrousos GP, et al. 1992. Mechanism of stress: A dynamic overview of hormonal and behavioural homeostasis. Neurosci Biobehav Rev, 16:115-30.

Kalueff AV, Tuohimaa P. 2005. Contrasting grooming phenotypes in three mouse strains markedly different in anxiety and activity (129S1, BALB/ C and NMRI). Behav Brain Res, 160:1-10.

Komorowska J, Pellis SM. 2004. Regulatory mechanisms underlying novelty-induced grooming in the laboratory rat. Behav Processes, 67:287-93.

Li S, Murakami Y, Wang M, et al. 2006. The effects of chronic valproate and diazepam in a mouse model of posttraumatic stress disorder. Pharmacol Biochem Behav, 85:324-31.

Liberzon I, Kirstov M, Young EA. 1997. Stress-Restress: Effects on ACTH and Fast Feedback. Psychoneuroendocrin, 22:443-53.

Louvart H, Maccari S, Ducrocq F, et al. 2005. Long-term behavioural alterations in female rats after a single intense footshock followed by situational reminders. Psychoneuroendocrin, 30:316-24.

McEwen BS. 2000. The neurobiology of stress: from serendipity to clinical relevance. Brain Res, 886:172-89.

Millan MJ, Girardon S, Dekeyne A. 1998. 5- $\mathrm{HT}_{2 \mathrm{C}}$ receptors are involved in the discriminative stimulus effects of citalopram in rats. Psychopharmacol, 142:432-4.

Nemeroff CB. 2004. Early - life adversity, CRF dysregulation, and vulnerability to mood and anxiety disorders. Psychopharmacol Bull, 38(Supp1 1):14-20.

Nico PB, De-Paris F, Vinade ER, et al. 2005. Altered behavioural response to acute stress in mice lacking cellular prion protein. Behav Brain Res, 162:173-81.

Ozer EJ, Best SR, Lipsey TL, et al. 2003. Predictors of posttraumatic stress disorder and symptoms in adults: a meta-analysis. Physiol Behav, 129:53-73.

Pellow S, Chopin P, File SE, et al. 1985. Validation of open:closed arm entries in an Elevated Plus-maze measurement of anxiety in the rat. J Neurosci Meth, 14:159-67.

Pfefferbaum B, Tucker P, North CS, et al. 2006. Persistent physiological reactivity in a pilot study of partners of firefighters after a terrorist attack. J Nerv Ment Dis, 194:128-31.

Pijlman FTA. 2001. Emotional and direct stress in rats. Doctoral thesis: Optima Grafische Communicatie, University of Utrecht, Nederland.

Prince CR, Anisman H. 1984. Acute and chronic stress effects on performance in a forced-swim task. Behav Neural Biol, 42:99-119.

Prut L, Belzung C. 2003. The open field as a paradigm to measure the effects of drugs on anxiety-like behaviors: a review. Euro J Pharmacol, 463(1-3):3-33.

Rodgers RJ, Dalvi A. 1997. Anxiety, defense and the elevated plus-maze. Neurosci Biobehav Rev, 21:801-10.

Schuster MA, Stein BD, Jaycox LH, et al. 2001. A National survey of stress reactions after the September 11, 2001, terrorist attacks. New Eng $J$ Med, 345:1507-12.

Stein BD, Elliot MN, Jaycox LH, et al. 2004. A National Longitudinal Study of the Indirect Consequences of the September 11, 2001 Terrorist Attacks: Reactions, Impairment, and Help-Seeking. Psychiatry, 67:105-17.

Uys JD, Marais L, Faure J, et al. 2006. Developmental trauma is associated with behavioral hyperarousal, altered HPA-axis activity, and decreased hippocampal neurotrophin expression in the adult rat. Ann NY Acad Sci, 1071:542-6.

Van den Berg CL, Lamberts R, Wolterink G, et al. 1998. Emotional and footshock stimuli induce differential long-lasting behavioural effects in rats; involvement of opoids. Brain Res, 799:6-15. 
Van der Hart MG, De Biurrun G, Czeh B, et al. 2005. Chronic psychosocial stress in tree shrews: effect of the substance $\mathrm{P}$ (NK1 receptor) antagonist L-760735 and clomipramine on endocrine and behavioural parameters. Psychopharmacol, 4:97-106.

Van Dijken HH, De Goeij DC, Sutano W, et al. 1993. Short inescapable stress produces long-lasting changes in the brain-pituitary-adrenal axis of adult male rats. Neuroendocrinol, 58:57-64.

Van Dijken HH, Mos J, Van der Heyden JA, et al. 1992. Characterization of stress induced long-term behavioral changes in rats: evidence in favor of anxiety. Physiol Behav, 52:945-51.
Van Erp AM, Kruk MR, Meelis W, et al. 1994. Effect of environmental stressors on time course, variability and form of self-grooming in the rat: handling, social contact, defeat, novelty, restraint and fur moistening. Behav Brain Res, 65:47-55.

Yehuda R, Southwick SM, Krystal JH, et al. 1993. Enhanced suppression of cortisol following dexamethasone administration in posttraumatic stress disorder. Am J Psychiatry, 150:83-6.

Zimering R, Gulliver SB, Knight J, et al. 2006. Posttraumatic stress disorder in disaster relief workers following direct and indirect trauma exposure to Ground Zero. J Trauma Stress, 19:553-7. 